\title{
TECHNIQUES FOR PREPARING HYDROGEL MEMBRANE CAPSULES
}

\author{
Somesh C. Nigam, I-Fu Tsao, Akiyoshi Sakoda and Henry Y. Wang* \\ Department of Chemical Engineering, University of Michigan \\ Ann Arbor, Michigan 48109, U.S.A.
}

\section{SUMMARY}

Techniques for preparing four kinds of hydrogel membrane capsules are described. In the present process the material being encapsulated remains in its original environment in an aqueous suspension. Also, capsule characteristics such as size, membrane thickness, pore-size and surface charge can be controlled over a wide range.

\section{INTRODUCTION}

Encapsulation techniques are finding increasing interest in a variety of areas of biotechnology. Enzymes, living cells, hormones, drugs, adsorbents and other bioactive materials can be encapsulated and used in bioreactors, artificial organs, controlled drug-release systems, bioseparation systems and etc (Chang, 1972; Kondo, 1979; Lim, 1984; Nigam, 1988; Nixon, 1976). Ideally, encapsulation of bioactive materials should be carried out in mild conditions and materials employed should be inert and non-toxic. However, a number of encapsulation techniques utilizing covalent binding hydrogel membranes require harsh conditions such as the use of non-aqueous solvents, extremes of $\mathrm{pH}$, or high temperature. Recently ionotropic gels have been used for encapsulating bioactive materials under mild conditions.

Lim et al. (1980; 1982; 1984) successfully encapsulated living cells in poly (L-lysine)-alginate capsules. Cells are initially entrapped in calcium-alginate gel beads. Calcium ions in the outer skin of the beads are displaced by poly (L-lysine) to form a polyelectrolyte complex membrane and calcium alginate gel thus wrapped by the complex is liquified using a calcium chelating agent. Difficulties in reproducing the complicated procedures of microencapsulation and in scale-up may restrict the extension of this method to a variety of applications. 
Spiekermann et al.(1987) and Klein et al. (1986) described a simplified method for encapsulating living cells in calcium-alginate capsules. In this method cells are not suspended in an alginate solution but in a calcium chloride solution. Droplets of calcium chloride solution containing cells are dropped into alginate solution and then instantly wrapped with calcium-alginate gel membrane. This method ensures that bioactive materials to be encapsulated never come into contact with the ionotropic gel-forming polymers and stay within their original environment. In addition to the simplicity of the procedures in comparison with the previous method (Lim et al., 1980; 1982; 1984), this method may also have the advantages of controlling and reproducing various characteristics of the hydrogel membrane such as thickness, pore-size, surface charge, mechanical properties and others by varying reaction time of capsule formation, alginate and/or calcium ion concentration(s) and employing different kinds of gel-forming polymer.

In this article techniques for preparing several new types of membrane capsules using different hydrogels are described. All of these techniques use mild conditions suitable for encapsulating even the most delicate bioactive materials such as animal cells. The capsules were formed using both single gel-forming polymer or a combination of several gel-forming polymers. Gel-forming polymers employed in this technique include alginate, agarose and chitosan. However the general methodology described can be easily extended to include a wide variety of other similar gel-forming polymers.

\section{MATERIALS AND METHODS}

Preparation of Calcium-Alginate Capsules: Calcium-alginate capsules were prepared using a simple one-step process similar to the one described by Spikerman et. al. (1987) and Klein et al. (1986). A solution containing $0.5 \%$ (w/v) sodium alginate (Kelco Gel LV) is prepared (Solution A). An another solution containing $1.3 \%$ (w/v) calcium chloride and 20\% (w/v) dextran (M.W. 40,000 ; Sigma) is prepared (Solution B), where dextran serves as a non-gelling polymer which is used to modulate the viscosity and density of Solution B to ensure spherical shape of capsules. It diffuses out of the capsule after the encapsulation is complete. Droplets of Solution B are dropped through a hypodermic needle into rapidly stirred Solution A. A capsular membrane forms instantaneously around the droplet due to the cross-linking of the interfacial alginate molecules by calcium cations. Prior to the removal of capsules Solution $\mathrm{A}$ is diluted more than five-fold by adding required amount of distilled water or appropriate buffer. This step dilutes the alginate solution outside the capsules and reduces the possibility of capsules joining each other when they are in close contact. The capsules are transferred to a $1.3 \%$ 
Preparation of Chitosan Capsules: Chitosan forms an ionotropic gel similar to the one formed by alginate. However chitosan is a cationic polymer and requires negatively charged multivalent ions (eg. polyphosphate ions) to form the gel structure. A solution containing $1.0 \%(\mathrm{w} / \mathrm{v})$ chitosan (Sigma) and $1.4 \%(\mathrm{v} / \mathrm{v})$ acetic acid is prepared (Solution $\mathrm{C}$ ). Also, a solution containing $1.5 \%(\mathrm{w} / \mathrm{v})$ sodium-tri-poly-phosphate solution and $40 \%(\mathrm{w} / \mathrm{v})$ dextran (Sigma) is prepared (Solution D). Droplets of the latter solution are dropped into Solution C. A chitosan polyphosphate membrane is instantly formed enclosing the droplet. Capsules are removed from the chitosan solution and further treated in $1.5 \%(\mathrm{w} / \mathrm{v})$ sodium-tri-poly-phosphate solution for half hour after washing away excess chitosan using $1.4 \%(\mathrm{v} / \mathrm{v})$ acetic acid.

Preparation of Alginate/Poly (L-lysine) Capsules: The basic approach to making capsules described in this paper can be adapted to produce the same type of capsule as proposed by Lim et al. $(1980 ; 1982 ; 1984)$. This not only simplifies the manufacturing procedure but also allows the bioactive material to remain in its original environment obviating the need to suspend it in the alginate solution. The exterior surface of calcium-alginate capsules prepared by the method described earlier is modified by treating with a $0.05 \%$ (w/v) poly (L-lysine) solution for 1 minute. The treated capsules are washed to remove uncomplexed poly (L-lysine).

Preparation of Agarose Capsules: In this preparation alginate is used as a template to form the agarose membrane. A solution containing $1.0 \%(w / v)$ agarose (Type VII, Sigma A) and $0.25 \%(w / v)$ sodium alginate (Type IV, Sigma) is prepared and kept around $40^{\circ} \mathrm{C}$ with stirring. An solution containing $1.3 \%(\mathrm{w} / \mathrm{v})$ calcium chloride and $20 \%(\mathrm{w} / \mathrm{v}$ ) dextran (Sigma) is also prepared. When the latter solution is dropped into the former solution, a capsular membrane is instantly formed around the droplet due to the cross-linking of the calcilim-alginate gel. The capsules thus formed are recovered and washed using prewarmed water at around $40^{\circ} \mathrm{C}$ to remove excess polymer solution. The capsules are subsequently cooled in water containing $1.3 \%(\mathrm{w} / \mathrm{v})$ calcium chloride which allows agarose entrapped within the membrane to solidify. The capsules are then equilibrated in a buffer containing $0.05 \mathrm{M}$ EDTA, which is calcium ion chelating agent, to liquify the alginate component of the membrane. Since agarose forms gels primarily through hydrogen bonding mechanism, these capsules remain stable in solutions containing high concentrations of $\mathrm{NaCl}$, EDTA, Phosphate etc. 


\section{RESULTS AND DISCUSSION}

The present process ensures that most of materials to be encapsulated never come in contact with the gel forming polymers, which suggests that this process is quite suitable for the encapsulation of bioactive materials. Toxic effects arising out of direct contact with charged polymers may affect the viability and growth of animal cells (Gharapetian et al.; 1986). Charged polyelectrolytes can also interfere with the functioning of bioactive materials such as enzymes by complexing with multivalent ions which may serve as cofactors.

Though calcium-alginate capsules can be produced by quite a simple procedure, in some applications capsules made of only calcium-alginate may not be suitable because of their instability in the presence of calcium sequestering agents such as phosphate, EDTA or high concentrations of monovalent cations. One way to overcome this problem is to modify these to alginate/poly (L-lysine) capsules or to form an entirely new type of gel capsule such as agarose capsules by the manner described in this article.

In a number of applications the capsule properties such as size, membrane thickness and pore-size may be manipulated to control the transport kinetics within the capsules. In the case of monoclonal antibody production using encapsulated hybridoma cells there may be advantages in selecting a pore-size which restricts the antibody within the capsule allowing the yield of antibody in a highly concentrated form (Lim, 1984). In our laboratory viable human carcinoma (KB) cells have been successfully encapsulated in using this method. Figure 1 shows a photomicrograph which demonstrites that encapsulated $\mathrm{KB}$ cells remain viable and show normal growth till tissue density. We are also able to grow monkey kidney cells (BSC-1) on microcarriers (Cytodex 1, Pharmacia) inside the calcium-alginate membrane capsules (Figure 2). Similarly applications involving encapsulated enzymes or antibodies require that the substrate or antigen may diffuse freely through the membrame while enzyme or antibody is retained inside the capsule. In applications for bioseparation fine particles of ion-exchange resins (DEAE Trisacryl) were encapsulated within calcium-alginate membrane and used to isolate and purify recombinan $\beta$-lactamase from crude $E$. coli homogenate (Nigam et al., 1988). Also. use of membrane encapsulated water-soluble polymer-ligand conjugates for hioseparation was described using Blue Dextran to isolate and purify human serum a!bumin (Figure 3) (Sakoda et al., 1988).

A number of capsule characteristics can be very easij cuntrolled using techniques described in this paper. The size of capsules can be varied from about $1(0)$ microns to at few millimeters in diameter by using a microdroplet generator in which the (rifice diameter and the air flow rate can be varied (Klein et al., 1983). 
The choice of membrane thickness should be carefully made to optimize its opposing effects on the capsule strength and the diffusional resistance. Also, the control of membrane thickness during the encapsulation process is essential for reproducing uniform capsules. In case of calcium-alginate capsules the thickness of gel membrane increases as the residence time of the droplet in the alginate solution increases due to the outward diffusion of calcium ion serving as a gelling inducer. The final membrane thickness is dependent on the capsule size, concentration of the gelling inducer and concentration of the polymer solution. By selecting appropriate conditions the membrane thickness can be very conveniently varied between $50 \mu \mathrm{m}$ to several hundred micrometers. The pore size of the membrane can be varied over a wide range of molecular weight cut-offs depending on the polymer employed and its concentration. Calcium-alginate gel in the range of 0.5 to $4 \%$ has a relatively smaller pore-size which can severely restrict the diffusion of proteins even in the molecular weight range of 60,000 to 100,000 (Tanaka et al.; 1984, Tramper; 1983) On the other hand agarose forms relatively porous gel in the range of 2 to $6 \%$, which allows minimal diffusional resistance even to the largest of enzymes (Tramper; 1983). By judicious choice of different polymers or their combinations it should be possible to tailor the capsule pore-size over a very wide range.

\section{ACKNOWLEDGEMENT}

We acknowledge the financial support from the National Science Foundation (CBT-8416543)

\section{REFERENCES}

Chang, T. M. S. (1972). Artificial Cells, Sprinfield; Charles C. Thomas.

Gharapetian, H., Davies, N. A. and Sun, A. M. (1986), Biotech. Bioeng., 28, $1595-1600$

Klein, J., Vorlop, K. D. and Steinert, H. J. (1986). German Patent DE 3432923.

Klein, J., Stock, J. and Vorlop, K. D. (1983). Eur. J. Appl. Microb. Biotech., 18, 86-91

Kondo, T. (1979). Microencapsulation, New Techniques and Applications, Tokyo; Techno Books

Lim, F., and Sun, A. M. (1980). Science, 210, 908-910

Lim, F. (1982). US Patent 4352883.

Lim, F. (1984). Biomedical Applications of Microencapsulation, Boca Raton; CRC Press.

Nigam, S. C., Sakoda, A. and Wang, H.Y., Biotech. Progress (in press)

Nixon, J. R. (1976). Microencapsulation, New York; Marcel Dekker.

Sakoda, A., Nigam, S. C. and Wang, H. Y. (1988). the 195-th ACS Annual Meeting, Toronto

Spiekermann, P., Vorlop, K. D., Klein, J. (1987). Proceedings of 4th European Congress on Biotechnology. Vol. 3. pp. 590-593.

Tanaka, H., Matsumura, M. and Veliky, I. A. (1984). Biotech. Bioeng, 26, 5358.

Tramper, J., in Solid Phase Biochemistry - Analytical and Synthetic Aspects, W. H. Scouten, Ed., Chapter 8; New York, John Willey and Sons 


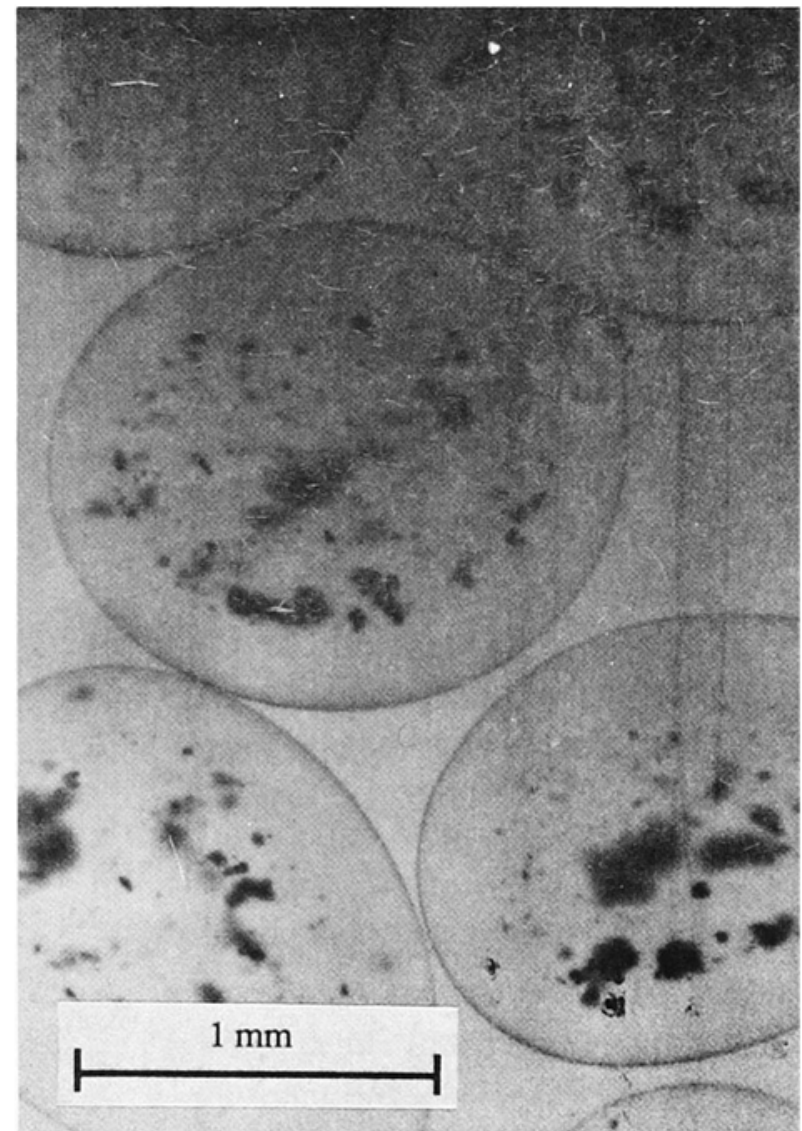

Figure 1 Grouth of $\mathrm{KB}$ cells in alginate/poly (L-lysine) capsules

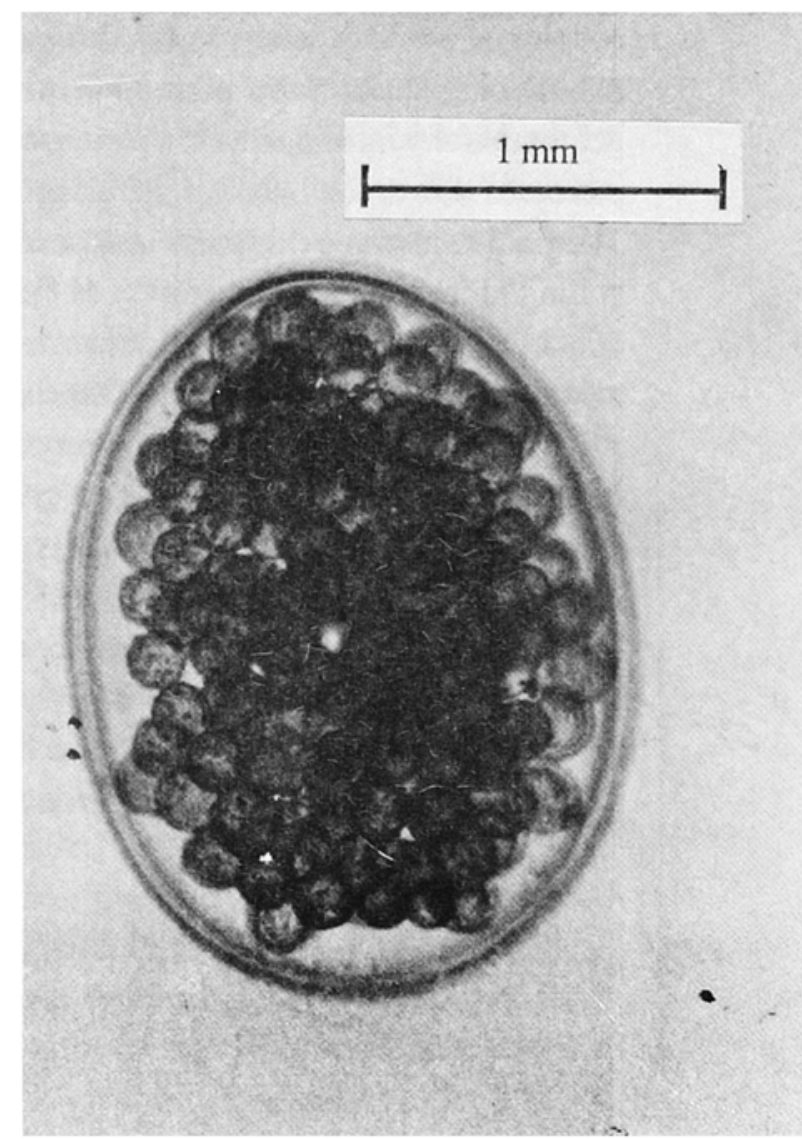

Figure 2 Monkey Kidney cells (BSC-1) on microcarriers inside a calcium-alginate capsle

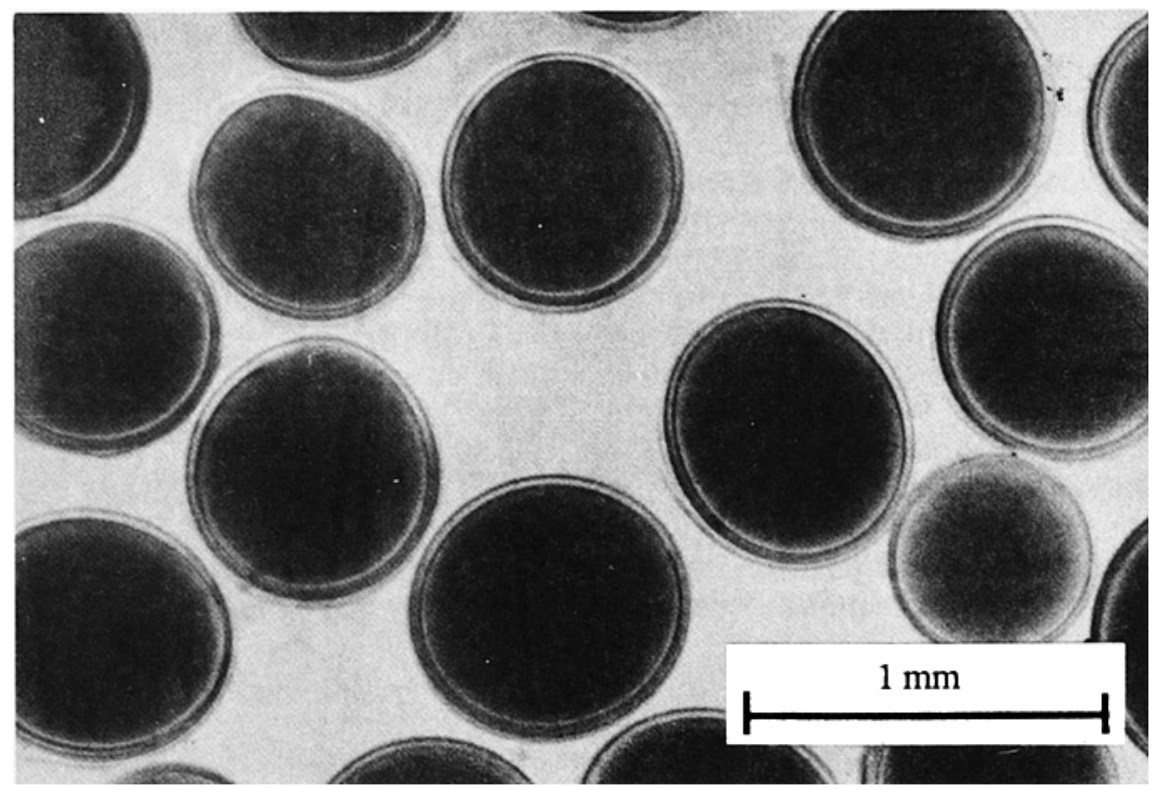

Figure 3 Uniform calcium-alginate capsules containing Blue Dextran solution 